\title{
Esm1 and Stc1 as Angiogenic Factors Responsible for Protective Actions of Adipose-Derived Stem Cell Sheets on Chronic Heart Failure After Rat Myocardial Infarction
}

\author{
Mai Watanabe, BSc; Hiromu Horie, MD; Yasutaka Kurata, MD, PhD; Yumiko Inoue, BSc; \\ Tomomi Notsu, BSc; Takayuki Wakimizu, BSc; Maya Adachi, BSc; Kenshiro Yamamoto, BSc; \\ Kumi Morikawa, PhD; Masanari Kuwabara, MD, PhD; Takuki Sakaguchi, MD; \\ Takayuki Morisaki, MD, PhD; Junichiro Miake, MD, PhD; Motonobu Nishimura, MD, PhD; \\ Motokazu Tsuneto, PhD; Yasuaki Shirayoshi, PhD; Shin Ito, MD, PhD; Masafumi Kitakaze, MD, PhD; \\ Haruaki Ninomiya, MD, PhD; Kazuhiro Yamamoto, MD, PhD; Ichiro Hisatome, MD, PhD
}

\begin{abstract}
Background: Although adipose-derived stem cell (ADSC) sheets improve the cardiac function after myocardial infarction (MI), underlying mechanisms remain to be elucidated. The aim of this study was to determine the fate of transplanted ADSC sheets and candidate angiogenic factors released from ADSCs for their cardiac protective actions.
\end{abstract}

Methods and Results: MI was induced by ligation of the left anterior descending coronary artery. Sheets of transgenic (Tg)-ADSCs expressing green fluorescence protein (GFP) and luciferase or wild-type (WT)-ADSCs were transplanted 1 week after MI. Both WT- and Tg-ADSC sheets improved cardiac functions evaluated by echocardiography at 3 and 5 weeks after MI. Histological examination at 5 weeks after Ml demonstrated that either sheet suppressed fibrosis and increased vasculogenesis. Luciferase signals from Tg-ADSC sheets were detected at 1 and 2 weeks, but not at 4 weeks, after transplantation. RNA sequencing of PKH (yellow-orange fluorescent dye with long aliphatic tails)-labeled Tg-ADSCs identified mRNAs of 4 molecules related to angiogenesis, including those of Esm1 and Stc1 that increased under hypoxia. Administration of Esm1 or Stc1 promoted tube formation by human umbilical vein endothelial cells.

Conclusions: ADSC sheets improved cardiac contractile functions after MI by suppressing cardiac fibrosis and enhancing neovascularization. Transplanted ADSCs existed for $>2$ weeks on MI hearts and produced the angiogenic factors Esm1 and Stc1, which may improve cardiac functions after Ml.

Key Words: Adipose-derived stem cell; Cell sheet; Esm1; RNA sequencing; Stc1

$\mathbf{T}$ he efficacy of pharmacological and surgical treatments for chronic heart failure after myocardial infarction (MI) is limited. Cell-based regenerative therapy (cell transplantation) improved the blood supply to the damaged heart, and minimized the area of infarc-

\begin{tabular}{l} 
Editorial p667 \\
\hline tion. $^{1-3}$ These beneficial effects are mediated, in part, by \\
cytokines, such as hepatocyte growth factor (HGF) and
\end{tabular}

cytokines, such as hepatocyte growth factor (HGF) and

Received August 19, 2020; revised manuscript received December 10, 2020; accepted January 11, 2021; J-STAGE Advance Publication released online March 12, 2021 Time for primary review: 25 days

Department of Genetic Medicine and Regenerative Therapeutics, Tottori University Graduate School of Medical Science, Yonago (M.W., H.H., Y.I., T.N., T.W., M.A., Ke. Yamamoto, M.T., Y.S., I.H.); Department of Physiology II, Kanazawa Medical University, Ishikawa (Y.K.); Biomaterials Research Group, Department of Life Science and Biotechnology, Health and Medical Research Institute, National Institute of Advanced Industrial Science and Technology, Tsukuba (K.M.); Intensive Care Unit and Department of Cardiology, Toranomon Hospital, Tokyo (M. Kuwabara); Division of Gastroenterology and Nephrology, Department of Multidisciplinary Internal Medicine (T.S.), Department of Pharmacology (J.M.), Division of Cardiovascular Surgery, Department of Surgery (M.N.), Department of Biological Regulation (H.N.), Division of Cardiovascular Medicine, Department of Molecular Medicine and Therapeutics (Ka. Yamamoto), Tottori University Faculty of Medicine, Yonago; Division of Molecular Pathology/Department of Internal Medicine IMSUT Hospital, The Institute of Medical Science, The University of Tokyo, Tokyo (T.M.); Department of Clinical Research and Development, National Cerebral and Cardiovascular Center, Suita (S.I.); and Hanwa Daini Senboku Hospital, Sakai (M. Kitakaze), Japan

These first two authors contributed equally to this work (M.W., H.H.).

Mailing address: Yasutaka Kurata, MD, PhD, Department of Physiology II, Kanazawa Medical University, 1-1 Daigaku, Uchinada, Kahoku, Ishikawa 920-0293, Japan. E-mail: yasu@kanazawa-med.ac.jp

All rights are reserved to the Japanese Circulation Society. For permissions, please e-mail: cj@j-circ.or.jp

ISSN-1346-9843 
vascular endothelial growth factor (VEGF), secreted by transplanted cells. ${ }^{4}$ However, direct injection of cells into the heart was problematic because of a significant loss of live cells and proarrhythmic effects. ${ }^{7,8}$ Tissue engineering using cell sheets has been developed to overcome these disadvantages; cell sheets could improve viability of cells 9,10 and prolong secretion of cytokines. ${ }^{11}$

Adipose-derived stem cells (ADSCs) are expected to be a useful cell source and ADSC sheets improve the cardiac function after experimental MI.12 ADSC sheets created by magnetite tissue engineering have been reported to improve the function of MI hearts. ${ }^{13}$ ADSCs secrete cytokines such as VEGF, HGF and fibroblast growth factor (FGF), and prevent cardiac dysfunction and remodeling after MI ${ }^{\mathbf{1 4}, 15}$ by inducing neovascularization. ${ }^{16,17}$ We have previously reported that ADSC sheets using temperature-responsive culture dishes decreased interstitial fibrosis, induced neovascularization, and prevented remodeling in rat MI hearts. ${ }^{12}$ Pretreatment with either the angiotensin receptor blocker (ARB), irbesartan, ${ }^{18}$ or a $\beta$-blocker, carvedilol, ${ }^{19}$ abolished neovascularization in hearts transplanted with ADSC sheets. These findings suggest that ADSC sheets could facilitate angiogenesis by secreting certain angiogenic factors, contributing to the improvement of MI heart function. However, it remains to be elucidated what types of angiogenic factors are expressed in ADSC sheets and how long transplanted ADSCs exist and secrete angiogenic factors.

In the present study, we determined the life-span of ADSC sheets transplanted into MI hearts and encompassed gene expressions of angiogenic factors in transplanted ADSC sheets. We found that transplanted ADSCs exist for more than 2 weeks and express 2 candidate molecules, endothelial cell specific molecule-1 (Esm1) and stanniocalcin-1 (Stc1), as angiogenic factors.

\section{Methods}

\section{Animal Care}

Adult male syngeneic Lewis rats weighing 200-250g (Japan SLC Inc., Hamamatsu, Japan) served as both donors and recipients of ADSC sheets. To determine the life-span of transplanted ADSC sheets, we developed dual reporter transgenic ( $\mathrm{Tg}$ ) rats in which green fluorescence protein (GFP) and luciferase are expressed through $\mathrm{P} 2 \mathrm{~A}$ alliance. ${ }^{20}$ The experimental protocols were approved by the Institutional Animal Care and Use Committee, Faculty of Medicine, Tottori University.

\section{Engineering of ADSC Sheets}

ADSCs were isolated from the inguinal subcutaneous fat tissue of 8-week-old wild-type (WT) Lewis rats and Tg rats of the same strain, and cultured as described previously. ${ }^{18,19}$ To prepare cell sheets, ADSCs were cultured on $35-\mathrm{mm}$ temperature-responsive culture dishes (UpCell, Cell Seed Inc., Tokyo, Japan) in an incubator at $37^{\circ} \mathrm{C}$ for $48 \mathrm{~h}$. They were then maintained at $20^{\circ} \mathrm{C}$ for $1 \mathrm{~h}$ and were detached as an intact sheet.

\section{Creation of a Rat MI Model and Transplantation of ADSC Sheets}

Rats were assigned to 4 groups: (1) no treatment after MI (Control MI group, n=7); (2) Tg-ADSC sheet transplantation 1 week after MI (Tg-ADSC group, n=9); (3) WTADSC sheet transplantation 1 week after MI (WT-ADSC group, $n=12$ ); and (4) sham operation without MI (shamoperated non-MI group, $n=10$ ). For the 3 MI groups, acute MI was induced by ligation of the left anterior descending artery, as described previously. ${ }^{12,18,19}$ Sheet transplantation, as described in our previous report, ${ }^{\mathbf{1 2}}$ was performed for the Tg-ADSC and WT-ADSC group rats 7 days after MI.

\section{Echocardiography}

Cardiac function was evaluated by echocardiography just before and 1, 3 and 5 weeks after MI ( 7 days before, just before, and 2 and 4 weeks after ADSC sheet transplantation) using a 12-MHz transducer (LOGIQ P5J and 12L; GE Healthcare, Fairfield, CT, USA). Short-axis 2-dimensional images at the mid-papillary level of the left ventricle (LV) were taken. The end-systolic and end-diastolic cavity areas were determined by tracing endocardial borders to calculate the fractional area shortening (FS). Left ventricular internal diameters at the end-systole (LVESD) and end-diastole (LVEDD) were measured between the mitral valve and papillary muscles to calculate the ejection fraction $(\mathrm{EF})$.

\section{Histopathology}

Five weeks after MI (4 weeks after transplantation), hearts were dissected, fixed in $10 \%$ formalin, and embedded in paraffin. Transverse sections obtained from the lateral wall, posterior wall and septum were subjected to Masson's trichrome staining to detect interstitial fibrosis. We determined the percentage of stained areas to the total sectional area by using a microscope (BZ9000; Keyence, Osaka, Japan). Each field was scanned, and digital images were analyzed by using Keyence analysis software. The sections were also stained with an anti-von Willebrand factor (vWF) antibody (Abcam plc, Cambridge, UK) to detect capillaries in the border zone between the myocardium and scar. The border zone is defined as the fibrotic area between the normal and infarcted areas, ${ }^{\mathbf{1 2 , 2 1}}$ whereas the remote zone as the normal area was localized on the opposite side of the infarction area. Capillaries were counted in 2 fields of transverse sections per animal. The number of capillaries in each field was averaged and expressed as capillary density.

\section{Ex Vivo Imaging of Langendorf Rat MI Hearts Transplanted With Tg-ADSC Sheets}

To see how long transplanted Tg-ADSC sheets exist on the surface of hearts, MI hearts were excised and subjected to the Langendorf experiment at 1, 2 and 4 weeks after transplantation. Isolation and coronary perfusion of hearts were conducted by using a Langendorff perfusion system (Model 7523-40; Masterflex, Barrington, IL, USA), as described previously. ${ }^{18}$ Coronary arteries were perfused with a modified Tyrode's solution $\left(\mathrm{NaCl} 144, \mathrm{KCl} 5, \mathrm{CaCl}_{2} 1.5\right.$, $\mathrm{MgCl}_{2}$ 0.9, HEPES 6, and D-glucose 5 [in mmol/L]; pH 7.4 with $\mathrm{NaOH}$ ) equilibrated with $100 \%$ oxygen. D-luciferin (Toyobo, Osaka, Japan) of $25 \mu \mathrm{mol}(1 \mathrm{~mL}$ of $25 \mathrm{mmol} / \mathrm{L}$ solution) was injected into coronary arteries of MI hearts through the aorta. Ten minutes after the D-luciferin injection into coronary arteries, bioluminescence images of Tg-ADSC sheet-transplanted MI hearts were obtained using the Xenogen IVIS 200 imaging system (Sumitomo, Tokyo, Japan).

\section{RNA Sequencing Analysis Using PKH (Yellow-Orange Fluorescent Dye With Long aliphatic Tails)-Positive ADSCs RNA sequence library preparation, sequencing, mapping,}



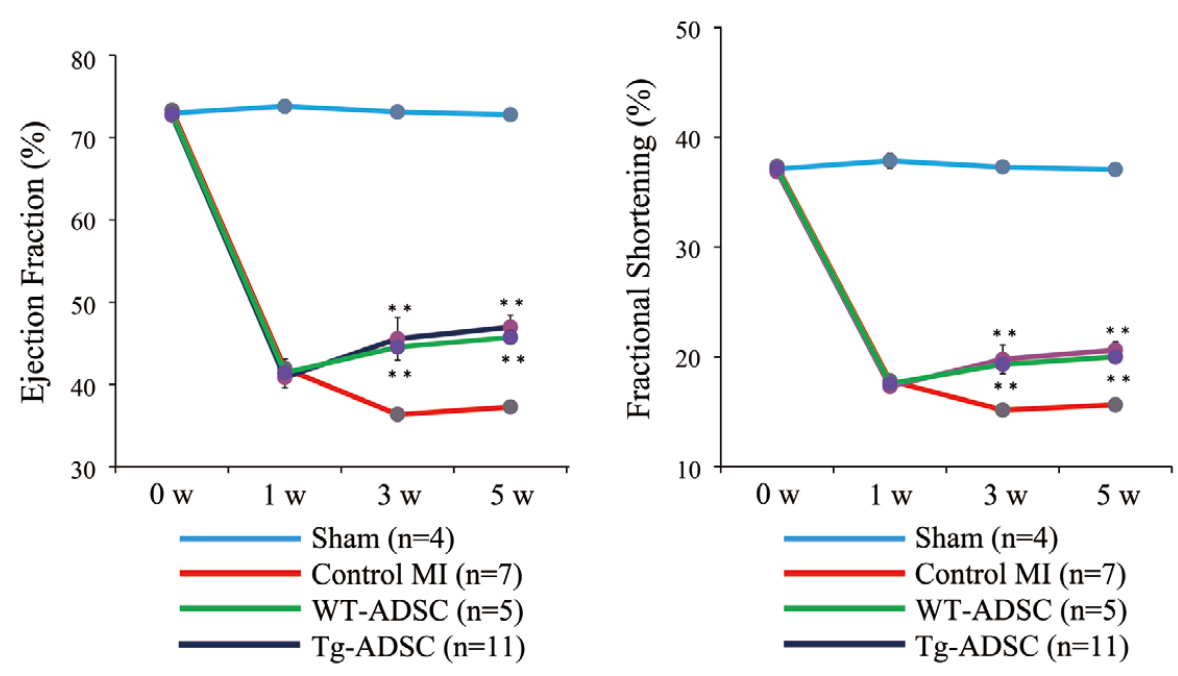

Figure 1. Effects of sheet transplantation of either Tg-ADSCs or WT-ADSCs on cardiac functions after MI. The ejection fraction (EF) and fractional area shortening (FS) of the hearts excised from rats are shown for the 4 groups. EF and FS were measured by echocardiography just before $\mathrm{Ml}(\mathrm{O}$ w), and 1 week $(1 \mathrm{w}), 3$ weeks $(3 \mathrm{w})$ and 5 weeks $(5 \mathrm{w})$ after $\mathrm{Ml}$; that is, 1 week before, just before, 2 weeks after and 4 weeks after transplantation of either a Tg- or WT-ADSC sheet for MI hearts. The numbers of experiments are given in the parentheses ( $n=4-11)$. ${ }^{\star \star} \mathrm{P}<0.01$ (vs. Control Ml group). Tg, transgenic; ADSC, adipose-derived stem cell; WT, wild type; MI, myocardial infarction.

gene expression analysis, and gene ontology (GO) enrichment analysis were performed by using DNAFORM (Yokohama, Japan). ${ }^{22}$ Qualities of total RNA were assessed by using a Bioanalyzer (Agilent, RNA 6000 kit; Tokyo, Japan) to ensure the RNA integrity number (RIN). RNA-seq libraries, which consist of double-stranded cDNA, were prepared by using a SMARTer Stranded Total RNA-seq kit-Pico Input Mammalian (Takara Bio, Shiga, Japan), following the manufacturer's instructions. The size and purity of cDNA libraries were confirmed by an Agilent 2100 Bioanalyzer (High Sensitivity DNA kit), and the libraries were quantified by quantitative polymerase chain reaction (PCR) using primer pairs that have complementary sequences of Illumina adapters (Illumina Inc., Tokyo, Japan). After denaturation, sequencing was conducted on Illumina HiSeq utilizing 150-bp paired-end reads. Obtained raw reads were trimmed and quality-filtered using Trim Galore! (ver. 0.4.4; Babraham Bioinformatics, Cambridge, UK), Trimmomatic (ver. 0.36), and cutadapt (ver. 1.16) software. Trimmed reads were then mapped to the rat genome (Rnor 6.0) using STAR (ver. 2.6.1a). Reads on annotated genes were counted using featureCounts (ver. 1.6.1). Fragments per kilobase of exon per million reads mapped (FPKM) values were calculated from mapped reads by normalizing to total counts and transcripts. Differentially expressed genes were detected by using the DESeq2 package (ver. 1.18.0). The list of differentially expressed genes detected by the DESeq2 package (basemean $>5$ and fold-change $<0.25$, or basemean $>5$ and fold-change $>4$ ) was used for GO enrichment analysis by the clusterProfiler package (ver. 3.6.0).

\section{Pathway Analysis}

The data obtained by RNA sequencing were loaded into Ingenuity pathway analysis software (Qiagen, Hilden, Germany) to identify functional relationships between molecules.

\section{Real-Time Reverse Transcription (RT)-PCR Analysis}

ADSCs were incubated at $37^{\circ} \mathrm{C}$ under normal $\left(21 \% \mathrm{O}_{2}\right)$ or hypoxic $\left(<2 \% \mathrm{O}_{2}\right)$ conditions for $48 \mathrm{~h}$ using an AnaeroPack system (MITSUBISHI GAS CHEMICAL Inc., Tokyo, Japan). Total RNA was extracted from ADSCs using the RNeasy Mini kit (QIAGEN inc., Valencia, CA, USA). Real-time RT-PCR analysis of VEGF, Esm1, Stc1, Ccbe1, Gng8, and $\beta$-actin was performed using the ABI 7900HT Fast Real-Time PCR System (Applied Biosystems, Foster City, CA, USA). Their mRNA levels were expressed as ratios to that of $\beta$-actin. The primers used are shown in the Supplementary Table. The mRNA levels were determined by using the comparative $\mathrm{Ct}$ method, and expressed as $2^{-\Delta \Delta \mathrm{Ct}}$.

\section{Tube Formation Assay}

Matrigel matrix growth factor reduced (GFR; BD Biosciences Japan, Tokyo, Japan) was thawed, dispensed in 24-well plates, and allowed to solidify at $37^{\circ} \mathrm{C}$ for $30 \mathrm{~min}$. Human umbilical vein endothelial cells (HUVECs) were seeded at a density of $5 \times 10^{4}$ cells per well, and incubated at $37^{\circ} \mathrm{C}$ for $1 \mathrm{~h}$. Then, a cell culture insert $(0.8 \mu \mathrm{m}$ pore; BD Biosciences Japan) was set on each well. HUVECs were resuspended in Dulbecco's Modified Eagle Medium (DMEM) and the inserts were removed after $18 \mathrm{~h}$. The network-like structures of HUVECs were examined under a microscope. Angiogenic activities were assessed by determining the total length of each tube.

\section{Data Analysis}

All the data are expressed as mean \pm SEM. Student's t-test and ANOVA with Tukey's post-hoc test were used for comparisons of the 2 groups and multiple $(\geq 3)$ groups, respectively. A probability value of $<0.05$ was considered significant. 
A

a) Control MI

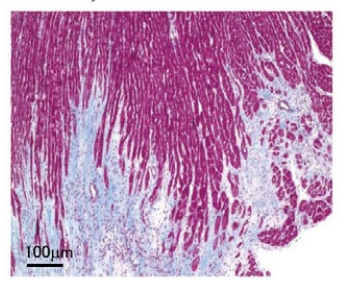

b) WT-ADSC

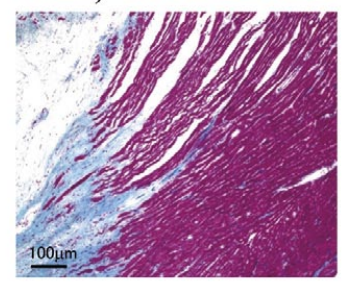

B

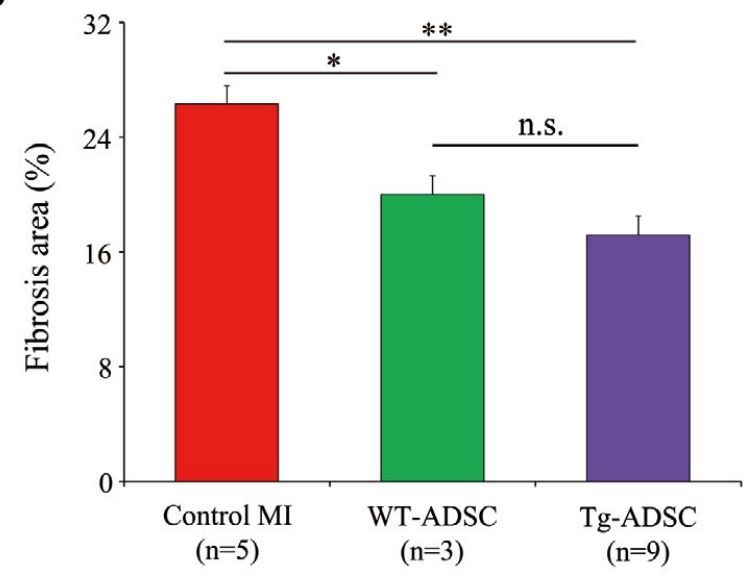

c) Tg-ADSC

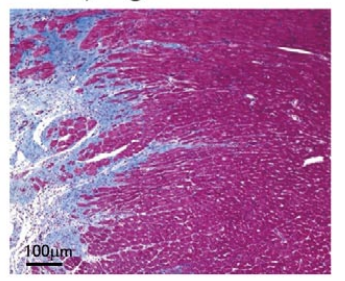

Figure 2. Effects of sheet transplantation of either Tg-ADSCs or WTADSCs on interstitial fibrosis in the border zone of MI hearts. (A) Representative pictures of interstitial fibrosis in $\mathrm{MI}$ hearts isolated from the Control MI (a), Tg-ADSC (b) and WT-ADSC (c) group rats 4 weeks after transplantation (5 weeks after MI). Picrosirius-red staining was performed to detect interstitial fibrosis. Scale bar $=100 \mu \mathrm{m}$. (B) Summary of the areas (\%) of interstitial fibrosis in the border zone of MI hearts from the 3 groups. The numbers of experiments are given in the parentheses for each group $(n=3-9)$. ${ }^{*}<<0.05$, ${ }^{*} \mathrm{P}<0.01$. Tg, transgenic; ADSC, adipose-derived stem cell; WT, wild type; MI, myocardial infarction; n.s., not significant.

\section{A}

a) Control MI

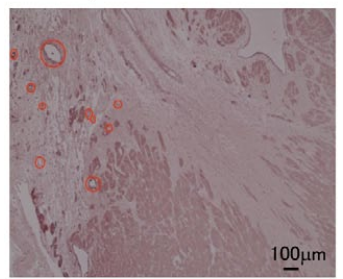

b) WT-ADSC

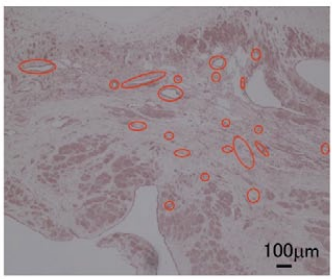

B

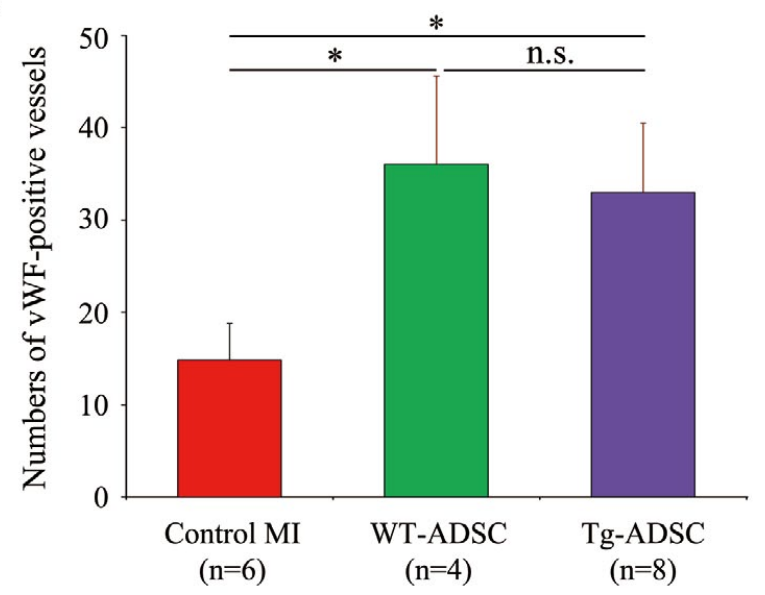

c) $\mathrm{Tg}-\mathrm{ADSC}$

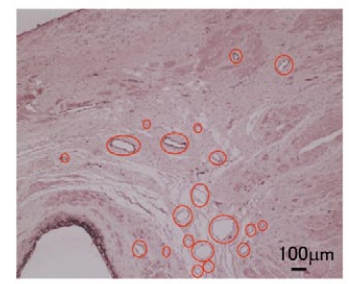

Figure 3. Effects of sheet transplantation of either Tg-ADSCs or WTADSCs on neovascularization in the border zone of Ml hearts. (A) Representative images of capillary vessels stained with an antibody against von Willebrand factor (VWF) in the border zone of $\mathrm{Ml}$ hearts isolated from the Control MI (a), Tg-ADSC (b) and WTADSC (c) group rats 5 weeks after Ml; that is, 4 weeks after ADSC sheet transplantation (orange ellipses; scale bar $=100 \mu \mathrm{m}$ ). (B) Summary of the numbers of vWF-positive vessels in the border zone of $\mathrm{Ml}$ hearts from each group. The number of capillary vessels was determined for 2 selected fields in the border zone per animal and expressed as an averaged value. The numbers of experiments are given in the parentheses $(n=4-8)$. ${ }^{\star} P<0.05$. Tg, transgenic; ADSC, adipose-derived stem cell; WT, wild type; MI, myocardial infarction; n.s., not significant. 


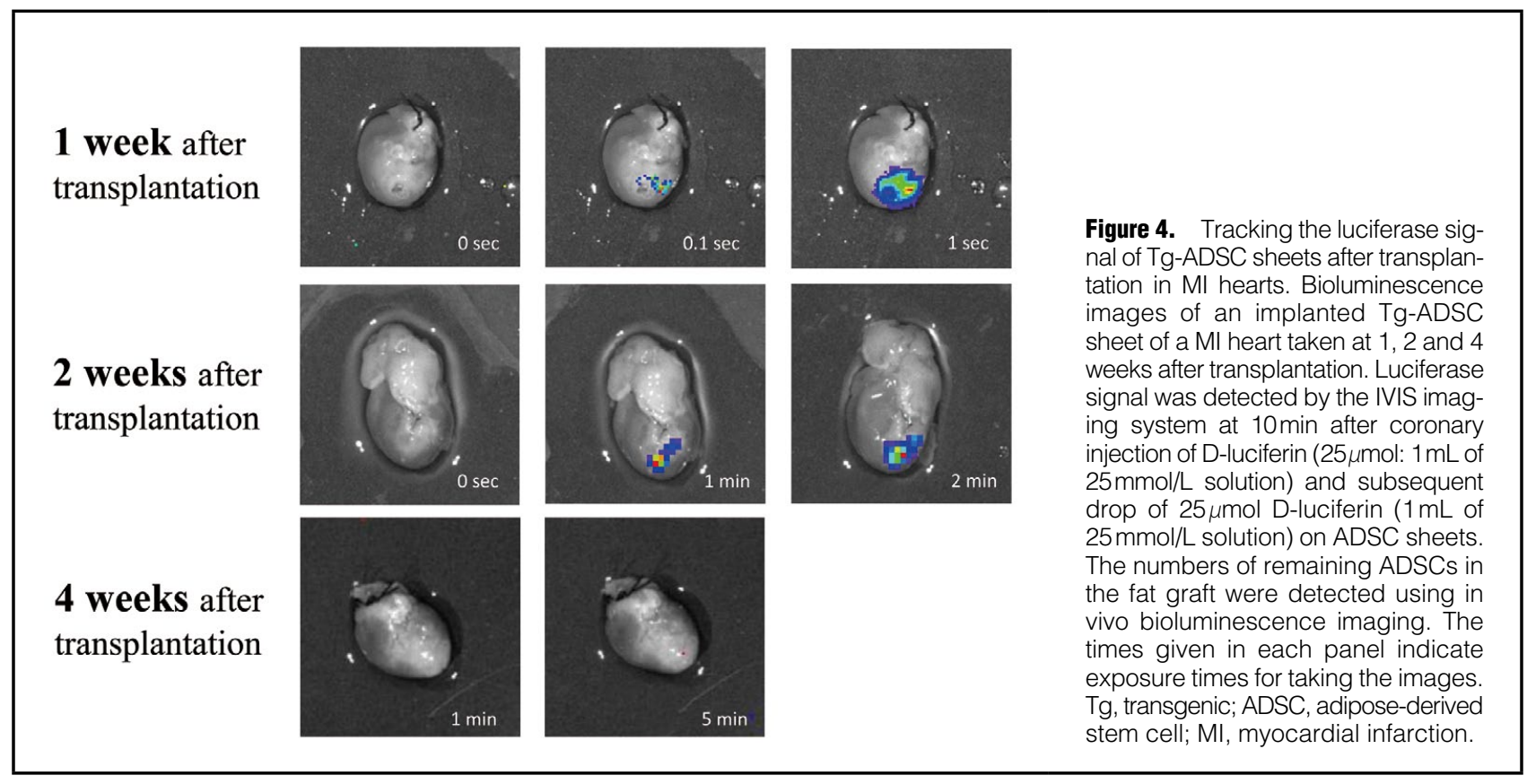

\begin{tabular}{|c|c|c|c|c|c|}
\hline Gene ID & Name & baseMean & log2FoldChange & IfcSE & padj \\
\hline ENSRNOG00000024536 & Ccbe1* & $1,225.483$ & 13.643 & 1.216 & 1.57203E-27 \\
\hline ENSRNOG00000010797 & Esm1* & $3,217.316$ & 13.195 & 1.055 & 4.92392E-34 \\
\hline ENSRNOG00000024843 & Prl8a9 & 300.43 & 11.615 & 1.251 & 4.59753E-19 \\
\hline ENSRNOG00000049695 & Myh2 & 277.556 & 11.501 & 1.226 & $1.96668 \mathrm{E}-19$ \\
\hline ENSRNOG00000015781 & Ndst3 & 265.65 & 11.438 & 1.229 & 3.66231E-19 \\
\hline ENSRNOG00000006653 & Slc38a4 & 246.443 & 11.329 & 1.254 & $4.38509 \mathrm{E}-18$ \\
\hline ENSRNOG00000020030 & Crlf1 & 226.377 & 11.207 & 1.241 & 4.54854E-18 \\
\hline ENSRNOG00000002537 & Wnk3 & 214.111 & 11.126 & 1.289 & 1.39559E-16 \\
\hline ENSRNOG00000021441 & Reln & 179.018 & 10.868 & 1.261 & 1.58955E-16 \\
\hline ENSRNOG00000015075 & Stc $1^{*}$ & 148.039 & 10.594 & 1.609 & $5.74405 \mathrm{E}-10$ \\
\hline ENSRNOG00000016701 & Gng8* & 143.069 & 10.544 & 1.265 & $1.62042 \mathrm{E}-15$ \\
\hline ENSRNOG00000008706 & Tbx3 & 141.839 & 10.532 & 1.263 & $1.59222 \mathrm{E}-15$ \\
\hline ENSRNOG00000014686 & Kcnd3 & 140.893 & 10.523 & 1.303 & $1.33301 \mathrm{E}-14$ \\
\hline ENSRNOG00000016099 & $\mathrm{Id} 4$ & 134.904 & 10.46 & 1.268 & 3.35369E-15 \\
\hline
\end{tabular}

baseMean, average of the normalized count values taken over all samples; log2FoldChange, log2 fold change between the groups; IfcSE, the standard error of the log2FoldChange estimate; padj, adjusted P value by using the Benjamini-Hochberg method. ${ }^{*}$ Candidates for angiogenic molecule genes are indicated.

\section{Results}

\section{Effects of Tg-ADSC Sheets on Cardiac Function, Fibrosis and Neovascularization of MI Hearts in Comparison to Those of WT-ADSC Sheets}

We first studied effects of Tg-ADSC and WT-ADSC sheets on cardiac functions and remodeling after $\mathrm{MI}$ in vivo. EF was significantly lower in the control MI group than in the shamoperated non-MI group. Two and four weeks after ADSC sheet transplantation ( 3 and 5 weeks after MI), EF and FS in the Tg-ADSC and WT-ADSC groups were significantly higher than those in the control MI group (Figure 1). There was no significant difference in the cardiac functions between the Tg-ADSC group and the WT-ADSC group. No rats died in any group during experiments (control MI group,
0/7; WT-ADSC group, 0/5; Tg-ADSC group, 0/11).

Cardiac fibrosis and neovascularization were evaluated by using histological analyses 4 weeks after transplantation (5 weeks after MI). In the control MI group, extensive fibrosis occurred around the infarcted region. This fibrosis was reduced in both the Tg-ADSC and WT-ADSC groups (Figure 2A). Figure 2B shows the summary data obtained from 3 to 9 experiments. The extent of cardiac fibrosis was significantly lower in both the Tg-ADSC and WT-ADSC groups than in the control MI group. There was no difference in cardiac fibrosis between the Tg-ADSC group and WT-ADSC group. The capillary density was remarkably increased in both the Tg-ADSC and WT-ADSC groups, as compared with that in the control MI group (Figure 3A). As summarized in Figure 3B, the capillary density was 


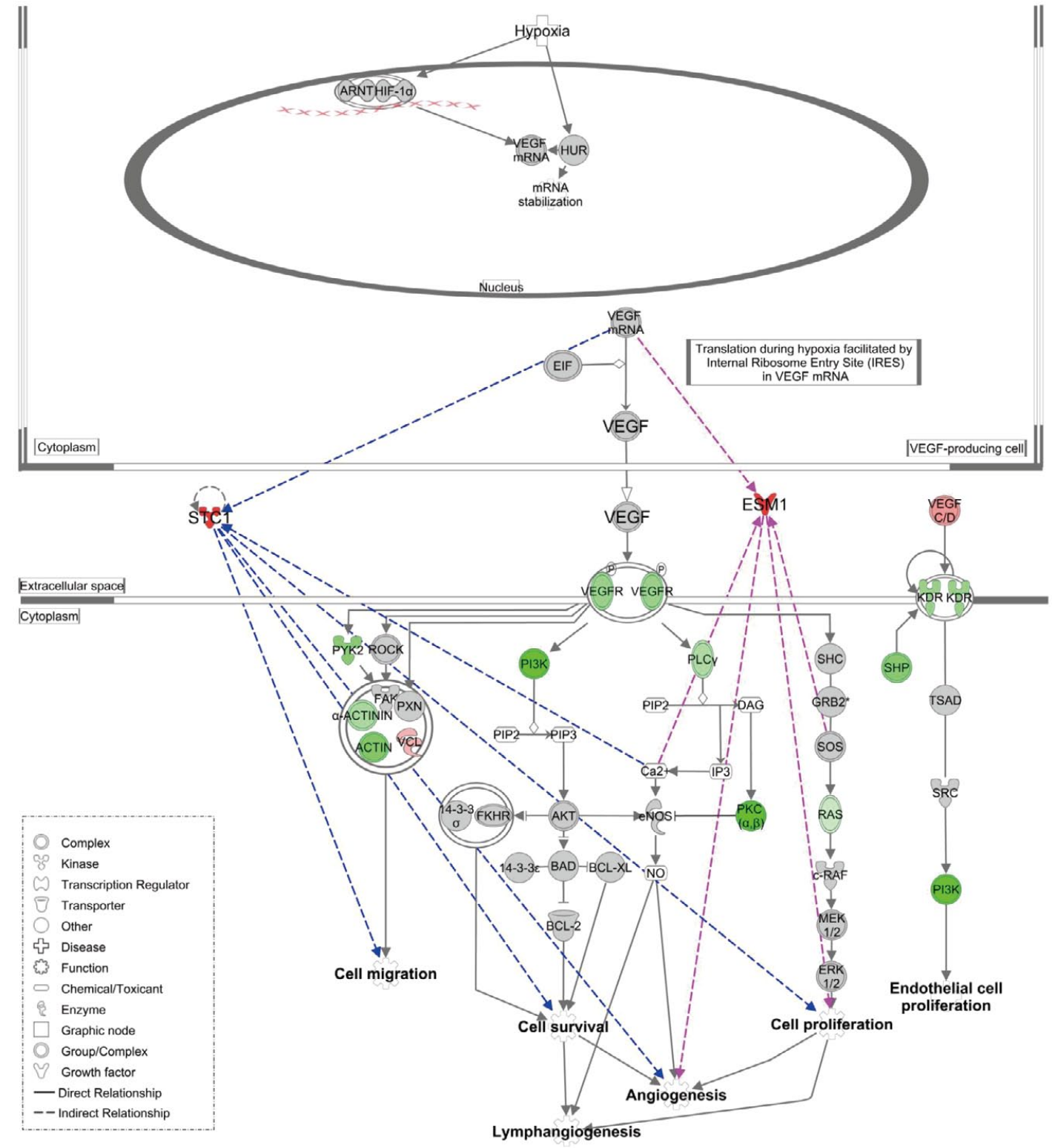

Figure 5. VEGF signaling canonical pathway constructed by Ingenuity Pathway Analysis. The schematic diagram shows the VEGF signaling pathway in PKH (yellow-orange fluorescent dye with long aliphatic tails)-positive ADSCs determined 1 week after transplantation (fold change $\geq 4 ; P<0.01$ ). Significantly upregulated and downregulated genes are shown in red and green, respectively. Grey color indicates the genes identified by RNA sequencing without changes in the expression level. Both Esm1 and Stc1 are predicted to enhance cell proliferation, cell migration, angiogenesis and cell survival, which are partially regulated by VEGF signaling. VEGF, vascular endothelial growth factor; ADSC, adipose-derived stem cell.

significantly higher in both the Tg-ADSC and WT-ADSC groups than in the control MI group. There was no difference in the capillary density between the Tg-ADSC group and WT-ADSC group. Thus, Tg-ADSC sheets enhanced neovascularization of MI hearts with a reduction of cardiac fibrosis to the same extent as WT-ADSC sheets.

\section{Tracking the Luciferase Signal of Tg-ADSC Sheets After Transplantation Into MI Hearts}

To evaluate survival of transplanted Tg-ADSC sheets, the signal of luciferase was determined by the IVIS imaging system 1, 2 and 4 weeks after Tg-ADSC sheet transplantation. Injection of D-luciferin into the coronary artery enabled visualization of the Enhanced Luc (ELuc) signal at the engraftment site by in vivo imaging (Figure 4). The ELuc signal was found in Tg-ADSC sheets in MI hearts at 1 and 2 weeks, but not 4 weeks after transplantation. This result suggests that transplanted Tg-ADSC sheets existed for more than 2 weeks (but disappeared within 4 weeks). 


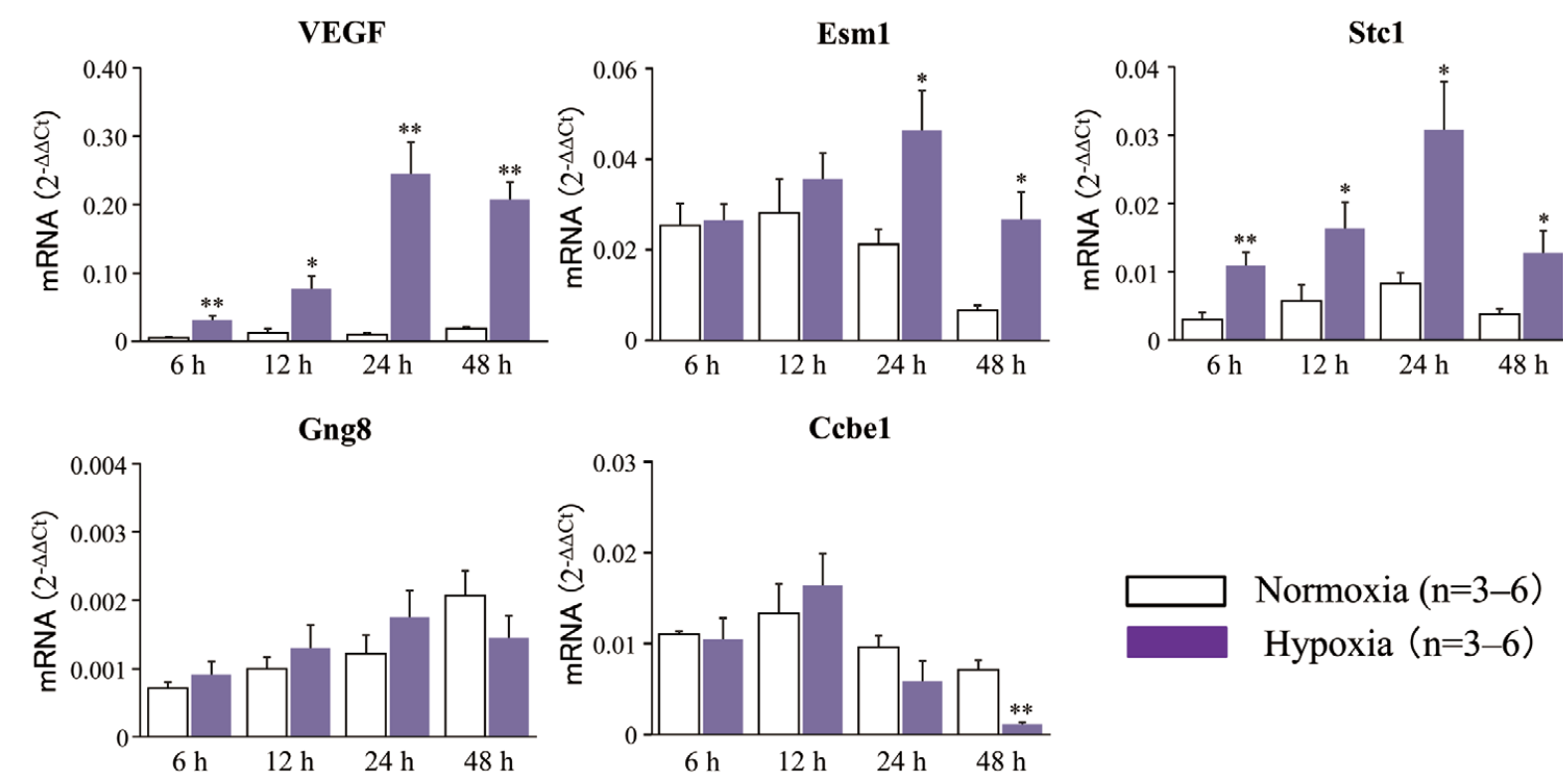

Figure 6. mRNA expression of candidate angiogenic molecules in ADSCs determined by RNA sequence analysis. Levels of Esm1, Stc1, Ccbe1 and Gng8 mRNAs as well as VEGF mRNA expressed in ADSCs were determined after incubation for 6, 12, 24 and $48 \mathrm{~h}$ under normoxia $\left(21 \% \mathrm{O}_{2}\right)$ or hypoxia $\left(<2 \% \mathrm{O}_{2}\right)$. Semi-quantitative RT-PCR analysis was performed to determine mRNA expression levels, which were normalized to $\beta$-actin mRNA levels. The numbers of experiments are given in the parentheses $(n=3-6) .{ }^{*} P<0.01,{ }^{*} P<0.05$ (vs. normoxia). ADSC, adipose-derived stem cell; Esm1, endothelial cell specific molecule-1; RT-PCR, reverse transcription polymerase chain reaction; Stc1, stanniocalcin-1; VEGF, vascular endothelial growth factor.

A

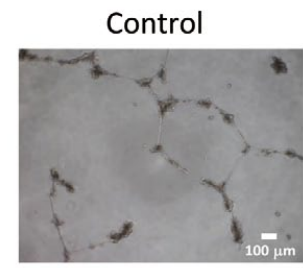

Esm1 $10 \mathrm{ng}$

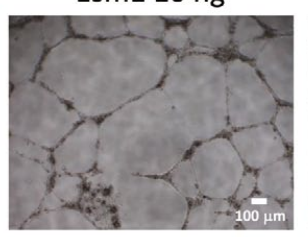

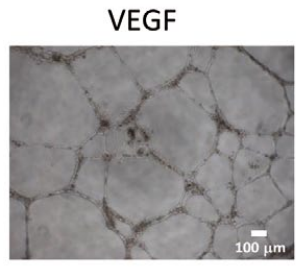

Stc1 $20 \mathrm{ng}$

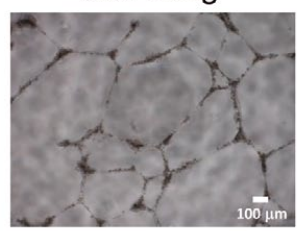

\section{B}

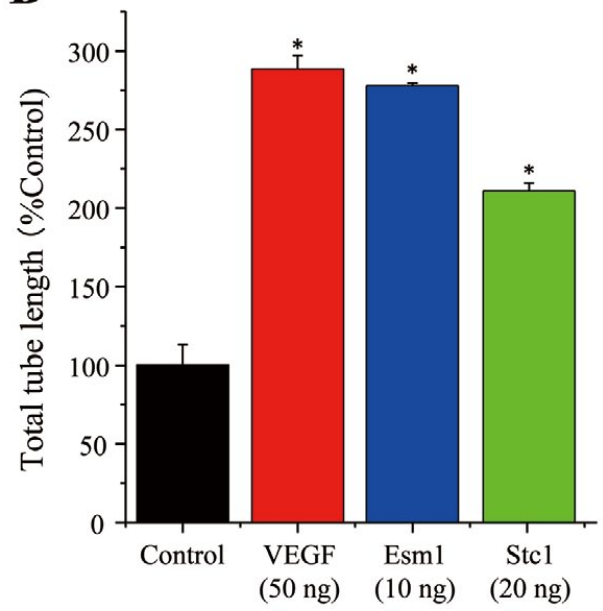

Figure 7. Effects of Esm1 and Stc1 on tube formation of HUVECs. (A) Representative images of the tube formation of HUVECs in the absence and presence of VEGF (50ng/well), Esm1 (10 ng/well) or Stc1 (20 ng/well) proteins. (B) The summary data on the total tube length obtained from multiple experiments of tube formation $(n=3-6)$. ${ }^{*} P<0.05$ (vs. Control). Esm1, endothelial cell specific molecule-1; Stc1, stanniocalcin-1; HUVECs, human umbilical vein endothelial cells; VEGF, vascular endothelial growth factor.

\section{Encompassing Analysis of Gene Expressions of Tg-ADSC Sheets Transplanted Into MI Hearts}

In order to encompass the gene expression of Tg-ADSC sheets, PKH-labeled Tg-ADSCs were enzymatically isolated from transplanted MI hearts at 1 week after transplantation and PKH-positive cells were sorted by fluorescence-activated cell sorting (FACS). Using PKH-positive cells, RNA sequencing was conducted to identify the mRNA expressed in TgADSCs. As shown in the Table, of top 15 genes expressed in PKH-positive cells, the 4 genes Esm1, Stc1, Ccbe1 and Gng8 
were picked up as the candidate genes related to angiogenesis. The pathway analysis indicates that both Esm1 and Stc1 are related to vascular angiogenesis (Figure 5).

To examine whether the upregulation of mRNA expressions of Esm1 and Stc1 occurs in the host MI heart (cardiomyocytes) transplanted with a ADSC sheet, we evaluated the mRNA expression of Esml and Stcl of the hearts in the control MI group and WT-ADSC group at 2 weeks after MI (1 week after transplantation). There was no significant difference in the mRNA level of Esm1 or Stc1 between the control MI group and WT-ADSC group (data not shown). This finding indicates that either Esm1 or STc1 mRNA expression do not increase in the host MI hearts transplanted with ADSC sheets, and that Esm1 and/or Stc1 proteins secreted from ADSCs play a pivotal role in promoting angiogenesis and improving cardiac functions after MI.

\section{Expression and Angiogenic Actions of Esm1 and Stc1 in ADSCs in Response to Hypoxia}

To examine changes in the transcription of candidate genes in response to hypoxia, we conducted a quantitative analysis of Esm1, Stc1, Ccbe1 and Gng8 mRNAs under normal and hypoxic conditions. As shown in Figure 6, Esm1 and Stc1 mRNAs as well as VEGF mRNA were significantly increased under hypoxic conditions, whereas Ccbel or Gng8 mRNA was not significantly altered. To determine the role of Esm1 and Stc1 in angiogenesis, it was examined whether recombinant Esm1 or Stcl proteins could promote in vitro angiogenesis of HUVECs as VEGF did. Figure 7A shows the representative tube formation of HUVECs in the absence and presence of VEGF, Esm1 or Stc1 proteins. Like VEGF, both Esm1 and Stc1 remarkably enhanced the tube formation of HUVECs. Figure 7B shows the summary data obtained from multiple experiments of tube formation. Administration of either Esm1 or Stc1 significantly increased the total tube length as the index of tube formation.

Besides, we studied the effect of conditioning medium from ADSCs transfected with siRNA against mRNA of either Esm1 or Stc1 on the tube formation of HUVECs. The conditioning medium from ADSCs with siRNA against Stc1 mRNA led to significantly reduced tube formation of HUVECs compared with that from the normal ADSCs, and that from ADSCs with siRNA against Esm1 mRNA tended to cause reduced tube formation (data not shown).

\section{Discussion}

In the present study, we found that transplantation of $\mathrm{Tg}$ ADSC sheets improved the cardiac function, increased angiogenesis, and reduced fibrosis after MI. Transplanted Tg-ADSC sheets existed at least for 2 weeks. Both Esm 1 and Stc1 mRNAs were significantly upregulated in ADSCs under hypoxic conditions. Administration of either Esm1 or Stc1 protein significantly facilitated the tube formation by HUVECs.

Tg-ADSC sheets improved cardiac functions of MI hearts as WT-ADSC sheets did. This finding indicates that neither GFP nor luciferase influences the cardioprotective actions of ADSC sheets. Tracking of the luciferase signal showed that the transplanted Tg-ADSC sheets existed for more than 2 weeks after transplantation, suggesting that the angiogenic factors released from ADSC sheets in the early stage after transplantation protect MI hearts against functional failure. It has been reported that transplanted ADSCs can differentiate into cardiomyocytes and endo- thelial cells in a MI model. ${ }^{23}$ However, immunofluorescence analyses indicated that implanted ADSCs did not differentiate into either endothelial cells or cardiac myocytes. ${ }^{12}$ ADSC therapy for MI repair has improved cardiac functions after MI through enhancement of angiogenesis via paracrine factors, ${ }^{24}$ and cardiac remodeling attenuation. $^{25}$ A previous study using ${ }^{124}$ I-HIB-labeled ADSCs visualized by positron emission tomography (PET) indicated that ADSCs transplanted into MI hearts survived only for 3 days after transplantation. ${ }^{26}$ Under a harsh microenvironment due to hypoxia in MI hearts, the viability of transplanted stem cells reduced due to apoptosis. ${ }^{27}$ In the present study, however, the luciferase signal of transplanted ADSCs obtained from the Tg-rat harboring the luciferase gene was observed in MI hearts for more than 2 weeks, which is far longer than the lifetime of the ${ }^{124}$ I-HIB signal of ADSCs. ${ }^{28}$ Taken together, transplanted ADSC sheets improved cardiac functions after MI through enhancement of angiogenesis and prevention of fibrosis, but not via their differentiation into cardiovascular cells. Because an $\mathrm{ARB}^{18}$ and a $\beta$-blocker ${ }^{19}$ abolished the ADSC sheet effects to restore cardiac functions of MI hearts, the angiogenic actions of ADSC sheets via the angiotensin receptor and $\beta$-adrenergic receptor play a pivotal role in the functional improvement of MI hearts. RNA sequence analysis for PKH-positive ADSCs collected from ADSC sheet-transplanted MI hearts showed the involvement of 4 candidate angiogenic factors, Ccbe1, Esm1, Stc1 and Gng8 in enhanced angiogenesis. Pathway analysis predicted Esm1 and Stc1 as angiogenic factors.

Esm1 named as Endocan is a dermatan sulfate proteoglycan expressed in endothelial cells, ${ }^{29}$ which is upregulated via either angiogenic factors such as VEGF-A or inflammatory cytokines such as tumor necrosis factor (TNF)- $\alpha .{ }^{30}$ Esm1 secreted from endothelial cells binds to VEGF on the cell surface, promoting the interaction of VEGF with VEGFR2 to enhance VEGF signaling. It has been reported that Esm1 binds to fibronectin and heparan sulfate proteoglycan 2, substitutes for fibronectin-bound VEGF-A165, and accelerates its bioavailability to promote angiogenesis through enhancement of the VEGF signal. ${ }^{31}$ Esm1 has also been reported to regulate phosphorylation of ERK1 and p38. ${ }^{32}$

Stanniocalcin (Stc) is a hypocalcemic glycoprotein secreted from the Stanius body and regulates homeostasis of both $\mathrm{Ca}^{2+}$ and phosphate in bony fishes. Stc1, a mammalian homologue of Stc, is known to be expressed in various tissues and is a downstream effector molecule of VEGF. ${ }^{33}$ It has been reported that Stcl facilitates the binding of VEGF to VEGFR2 to enhance neovascularization, and increases VEGFR2 in the retina. ${ }^{34}$ Taken together with the present RNA sequencing data, Esm1 and Stc1 released from transplanted ADSC sheets may act as angiogenic factors to induce neovascularization of MI hearts.

In the present study, hypoxic conditions could upregulate the mRNA expression of both Esm1 and Stc1, as well as VEGF in ADSCs. Both recombinant Esm1 and Stc1 significantly increased tube formation of HUVECs in vitro. Thus, hypoxic conditions may increase Esm1 and Stc1 released from ADSCs, leading to the tube formation of endothelial cells. Pathway analysis also showed that the hypoxic condition of ADSC sheets could activate expressions of Esm1 and Stc1. Conditioning medium from ADSCs with siRNA against Stc1 led to significantly decreased tube formation of HUVECs. This result suggests that $\mathrm{Stc1}$ plays a central role in ADSC-induced angiogenesis, 
whereas both Esm1 and Stc1 may be involved in the ADSC sheet enhancement of angiogenesis. In our study, either HGF or FGF2 mRNA in ADSCs did not significantly increase under the hypoxic condition for $24 \mathrm{~h}$ (data not shown, but see reference 12). These findings in vitro may be extrapolated to the status in vivo, suggesting that ischemic conditions of transplanted ADSC sheets in MI hearts could stimulate the secretion of the angiogenic factors, Esm1 and Stc1.

Reasons for the lack of enhanced expression of Ccbe1 and Gng8 in ADSCs under the hypoxic condition remain unknown. Ccbel has been reported to enhance lymphangiogenesis through the VEGF-C - VEGF Receptor 3 (VEGFR3) pathway, ${ }^{35}$ which may not be involved in the angiogenesis stimulated by ADSCs. Gng8 participates in the multi-cell signal transduction through the cell membrane G-protein phosphorylation and dephosphorylation. ${ }^{36}$ Although a previous report indicated that Gng8 was associated with the ability of inflammatory zone 1 (FIZZ1) to promote rat aortic endothelial cell (RAEC) in vitro angiogenesis, ${ }^{37}$ our study suggests that Gng8 is not implicated in the enhanced angiogenesis by ADSCs in MI hearts under hypoxic conditions.

In the present study, there were several limitations. First, we did not measure the infarct size of the control MI, WT$\mathrm{ADSC}$ or $\mathrm{Tg}-\mathrm{ADSC}$ groups. However, the values of $\mathrm{EF}$ in the 3 groups were approximately equal to those found in our previous reports.12,18,19 Given that the infarct sizes of the 3 groups were proportional to their values of EF, the infarct sizes of the 3 groups in this study were comparable to those found in our previous studies. Second, the effectiveness of the blank sheet was not examined in the present study. Nevertheless, we previously evaluated the effects of dermal fibroblast sheets as the blank sheet on contractile functions of MI hearts and demonstrated that the function of MI hearts transplanted with dermal fibroblast sheets was comparable to that of the control group MI hearts without transplantation. ${ }^{21}$ Finally, we examined the mRNA expression profile of transplanted ADSC sheets only 7 days after transplantation; the mRNA expression profile could not be determined in an earlier phase of transplantation because of the limited number of collected ADSCs in that phase. Moreover, we did not directly examine whether the transplantation of ADSC sheets that express neither Esm1 nor Stc1 could exert their protective effects on MI hearts as observed in this study.

Despite these limitations, the present study clearly shows that the transplanted ADSC sheet, existing on MI hearts for more than 2 weeks, improves the cardiac function after MI via increased expressions of the angiogenic factors, Esm1 and Stc1.

\section{Disclosures}

I.H. reported receiving lecturer's fee from Mochida Pharmaceutical Company, Sanwa Kagaku Kenkyusho Co. Ltd, Teijin Pharma Co. Ltd. and Fuji Yakuhin Co. Ltd., and research grants from Mochida Pharmaceutical Company, Teijin Pharma, Fuji Yakuhin Co. Ltd and Sanwa Kagaku Kenkyusho Co. Ltd.

M. Kitakaze, K. Yamamoto, and I.H. are members of Circulation Journal's Editorial Team.

\section{IRB Information}

The present study was approved by the Institutional Animal Care and Use Committee, Faculty of Medicine, Tottori University (Reference number: $15-\mathrm{Y}-51,17-\mathrm{Y}-16)$.

\section{References}

1. Kawamura M, Miyagawa S, Miki K, Saito A, Fukushima S, Higuchi T, et al. Feasibility, safety, and therapeutic efficacy of human induced pluripotent stem cell-derived cardiomyocyte sheets in a porcine ischemic cardiomyopathy model. Circulation 2012; 126: S29-S37.

2. Menasché $\mathbf{P}$, Alfieri O, Janssens $\mathrm{S}, \mathrm{McKenna} \mathrm{W}$, Reichenspurner $\mathrm{H}$, Trinquart $\mathrm{L}$, et al. The Myoblast Autologous Grafting in Ischemic Cardiomyopathy (MAGIC) trial: First randomized placebocontrolled study of myoblast transplantation. Circulation 2008; 117: $1189-1200$.

3. Bolli R, Tang XL, Sanganalmath SK, Rimoldi O, Mosna F, Abdel-Latif A, et al. Intracoronary delivery of autologous cardiac stem cells improves cardiac function in a porcine model of chronic ischemic cardiomyopathy. Circulation 2013; 128: 122-131.

4. Zhao T, Zhao W, Meng W, Liu C, Chen Y, Gerling IC, et al. VEGF-C/VEGFR-3 pathway promotes myocyte hypertrophy and survival in the infarcted myocardium. Am J Transl Res 2015; 7: $697-709$.

5. Rong SL, Wang XL, Zhang CY, Song ZH, Cui LH, He XF, et al. Transplantation of HGF gene-engineered skeletal myoblasts improve infarction recovery in a rat myocardial ischemia model. PLoS One 2017; 12: $\mathrm{e} 0175807$.

6. Liu J, Wu P, Wang Y, Du Y, Nan A, Liu S, et al. Ad-HGF improves the cardiac remodeling of rat following myocardial infarction by upregulating autophagy and necroptosis and inhibiting apoptosis. Am J Transl Res 2016; 8: 4605-4627.

7. Raedschelders K, Ansley DM, Chen DD. The cellular and molecular origin of reactive oxygen species generation during myocardial ischemia and reperfusion. Pharmacol Ther 2012; 133: $230-255$.

8. Song H, Cha MJ, Song BW, Kim IK, Chang W, Lim S, et al. Reactive oxygen species inhibit adhesion of mesenchymal stem cells implanted into ischemic myocardium via interference of focal adhesion complex. Stem Cells 2010; 28: 555-563.

9. Hamdi H, Planat-Benard V, Bel A, Puymirat E, Geha R, Pidial $\mathrm{L}$, et al. Epicardial adipose stem cell sheets results in greater postinfarction survival than intramyocardial injections. Cardiovasc Res 2011; 91: 483-491.

10. Patila T, Miyagawa S, Imanishi Y, Fukushima S, Siltanen A, Mervaala E, et al. Comparison of arrhythmogenicity and proinflammatory activity induced by intramyocardial or epicardial myoblast sheet delivery in a rat model of ischemic heart failure. PLoS One 2015; 10: e0123963.

11. Memon IA, Sawa Y, Fukushima N, Matsumiya G, Miyagawa S, Taketani S, et al. Repair of impaired myocardium by means of implantation of engineered autologous myoblast sheets. J Thorac Cardiovasc Surg 2005; 130: 1333-1341.

12. Otsuki Y, Nakamura Y, Harada S, Yamamoto Y, Ogino K, Morikawa K, et al. Adipose stem cell sheets improved cardiac function in the rat myocardial infarction, but did not alter cardiac contractile responses to $\beta$-adrenergic stimulation. Biomed Res 2015; 36: 11-19.

13. Ishii M, Shibata R, Shimizu Y, Yamamoto T, Kondo K, Inoue Y, et al. Multilayered adipose-derived regenerative cell sheets created by a novel magnetite tissue engineering method for myocardial infarction. Int J Cardiol 2014; 175: 545-553.

14. Sekiya N, Matsumiya G, Miyagawa S, Saito A, Shimizu T, Okano T, et al. Layered implantation of myoblast sheets attenuates adverse cardiac remodeling of the infarcted heart. $J$ Thorac Cardiovasc Surg 2009; 138: 985-993.

15. Gnecchi M, Zhang Z, Ni A, Dzau VJ. Paracrine mechanisms in adult stem cell signaling and therapy. Circ Res 2008; 103: 1204-1219.

16. Harada Y, Yamamoto Y, Tsujimoto S, Matsugami H, Yoshida A, Hisatome I. Transplantation of freshly isolated adipose tissuederived regenerative cells enhances angiogenesis in a murine model of hind limb ischemia. Biomed Res 2013; 34: 23-29.

17. Rehman J, Traktuev D, Li J, Merfeld-Clauss S, Temm-Grove CJ, Bovenkerk JE, et al. Secretion of angiogenic and antiapoptotic factors by human adipose stromal cells. Circulation 2004; 109: $1292-1298$.

18. Yamamoto K, Kurata Y, Inoue Y, Adachi M, Tsuneto M, Miake J, et al. Pretreatment with an angiotensin II receptor blocker abolished ameliorating actions of adipose-derived stem cell sheets on cardiac dysfunction and remodeling after myocardial infarction. Regen Ther 2018; 9: 79-88.

19. Adachi M, Watanabe M, Kurata $Y$, Inoue $Y$, Notsu T, Yamamoto $\mathrm{K}$, et al. $\beta$-Adrenergic blocker, carvedilol, abolishes ameliorating actions of adipose-derived stem cell sheets on cardiac dysfunc- 
tion and remodeling after myocardial infarction. Circ J 2019; 83: $2282-2291$.

20. Morikawa K, Nakamura K, Suyama Y, Yamamoto K, Fukuoka $\mathrm{K}$, Yagi S, et al. Novel dual-reporter transgenic rodents enable cell tracking in animal models of stem cell transplantation. Biochem Biophys Rep 2019; 18: 100645.

21. Harada S, Nakamura Y, Shiraya S, Fujiwara Y, Kishimoto Y, Onohara T, et al. Smooth muscle cell sheet transplantation preserve cardiac function and minimize cardiac remodeling in a rat myocardial infarction model. J Cardiothorac Surg 2016; 11: 131.

22. Kato M, Hamazaki Y, Sun S, Nishikawa Y, Kage-Nakadai E. Clostridium butyricum MIYAIRI 588 increases the lifespan and multiple-stress resistance of Caenorhabditis elegans. Nutrients 2018; 10: 1921.

23. Lin F. Adipose tissue-derived mesenchymal stem cells: A fat chance of curing kidney disease? Kidney Int 2012; 82: 731-733.

24. Mozid AM, Arnous S, Sammut EC, Mathur A. Stem cell therapy for heart diseases. Br Med Bull 2011; 98: 143-159.

25. Russo V, Young S, Hamilton A, Amsden BG, Flynn LE. Mesenchymal stem cell delivery strategies to promote cardiac regeneration following ischemic injury. Biomaterials 2014; 35: 3956-3974.

26. Kim MH, Woo SK, Lee KC, An GI, Pandya D, Park NW, et al. Longitudinal monitoring adipose-derived stem cell survival by PET imaging hexadecyl-4-[ $\left.{ }^{124} \mathrm{I}\right]$-iodobenzoate in rat myocardial infarction model. Biochem Biophys Res Commun 2015; 456: 13-19.

27. Penna C, Raimondo S, Ronchi G, Rastaldo R, Mancardi D, Cappello S, et al. Early homing of adult mesenchymal stem cells in normal and infarcted isolated beating hearts. $J$ Cell $\mathrm{Mol} \mathrm{Med}$ 2008; 12: 507-521.

28. Rodriguez-Porcel M, Gheysens O, Chen IY, Wu JC, Gambhir SS. Image-guided cardiac cell delivery using high-resolution small-animal ultrasound. Mol Ther 2005; 12: 1142-1147.

29. Hughes CC. Identification of endothelial cell genes expressed in an in vitro model of angiogenesis: Induction of ESM-1, (beta)igh3, and NrCAM. Microvasc Res 2002; 63: 159-171.

30. Roudnicky F, Poyet C, Wild P, Krampitz S, Negrini F, Huggenberger $\mathrm{R}$, et al. Endocan is upregulated on tumor vessels in invasive bladder cancer where it mediates VEGF-A induced angiogenesis. Cancer Res 2013; 73: 1097-1106.

31. Brutsch R, Liebler SS, Wustehube J, Bartol A, Herberich SE, Adam MG, et al. Integrin cytoplasmic domain-associated protein-1 attenuates sprouting angiogenesis. Circ Res 2010; 107: $592-601$.

32. Su T, Zhong Y, Demetriades AM, Shen J, Sui A, Yao Y, et al. Endocan blockade suppresses experimental ocular neovascularization in mice. Invest Ophthalmol Vis Sci 2018; 59: 930-939.

33. Simons M, Gordon E, Claesson-Welsh L. Mechanisms and regulation of endothelial VEGF receptor signaling. Nat Rev Mol Cell Biol 2016; 17: 611-625.

34. Law AY, Wong CK. Stanniocalcin-1 and -2 promote angiogenic sprouting in HUVECs via VEGF/VEGFR2 and angiopoietin signaling pathways. Mol Cell Endocrinol 2013; 374: 73-81.

35. Weijts BG, van Impel A, Schulte-Merker S, de Bruin A. Atypical E2fs control lymphangiogenesis through transcriptional regulation of Ccbel and Flt4. PLoS One 2013; 8: e73693.

36. Ryba NJ, Tirindelli R. A novel GTP-binding protein gammasubunit, G gamma 8, is expressed during neurogenesis in the olfactory and vomeronasal neuroepithelia. J Biol Chem 1995; 270: $6757-6767$.

37. Li X, Yang Y, Fang J, Zhang H. FIZZ1 could enhance the angiogenic ability of rat aortic endothelial cells. Int J Clin Exp Pathol 2013; 6: 1847-1853.

\section{Supplementary Files}

Please find supplementary file(s);

http://dx.doi.org/10.1253/circj.CJ-20-0877 